\title{
Using Confusion Graphs to Understand Classifier Error
}

\author{
Davis Yoshida and Jordan Boyd-Graber \\ davis.yoshida@colorado.edu, jordan.boyd.graber@colorado.edu
}

\begin{abstract}
Understanding the nature of the errors of a machine learning system is often difficult for multiclass classification problems with a large number of classes. This is true even more so if the number of examples for each class is low. To interpret the performance of a multiclass classifier, we form a graph representing the errors, and use average-link clustering to find groups of classes which are confused with each other. We apply this idea to the QANTA question answering system (Iyyer et al., 2014), and provide a method of analysis of the clusters.
\end{abstract}

\section{Introduction}

A typical development process for a machine learning pipeline involves many iterations of error analysis, and changes to each step of the pipeline. One of the most common tools for understanding the errors of multiclass classifiers is a confusion matrix. If, however, the number of classes is very large relative to the number of samples, the confusion matrix will be both large and sparse. This is problematic for error analysis, because adding a feature which reduces the confusion between two classes amounts to decreasing the overall error by a tiny increment. If the error is attacked on a class by class basis, the process will be both very lengthy and result in features which may not discriminate between any pairs of classes other than the one they were created for. While it is certainly possible to engineer features which are widely useful, it would be beneficial to do so this both consistently and quickly.
One example of a multiclass classification system with a large number of classes is QANTA, a factoid question answering system. QANTA is designed to play the Quiz Bowl trivia game, in which players are given questions word by word, and want to answer the question correctly as early as possible. Since the number of answers QANTA is trained on is finite, it can be viewed as a multiclass classifier with a large number of classes. For any given pair of answers, it is highly unlikely that there are more than a few questions for which the system confused one answer with the other. This makes QANTA a perfect example of a multiclass classifier which has a sparse confusion matrix. To better analyze the errors committed by the system we form a confusion graph for each of three subsets of our data. The construction of these graphs and some of their interesting properties are discussed in Section 2.

In Section 3, we discuss the use of average-link agglomerative clustering to find clusters of answers which are highly confused with each other on a validation set.

In Section 4, we apply the Autoslog system (Riloff, 1996) to each cluster to find find textual features which occur frequently in each cluster.

\section{The Confusion Graph}

After training QANTA, we use the errors it commits on a validation set to form the confusion graph. In this graph, the vertices are answers (classes), and the undirected weighted edges represent errors. Specifically, an edge $(u, v)$ with weight $w$ means that the fraction of questions having $u$ as their correct answer while the system predicted $v$, or vice versa, is $w$. 


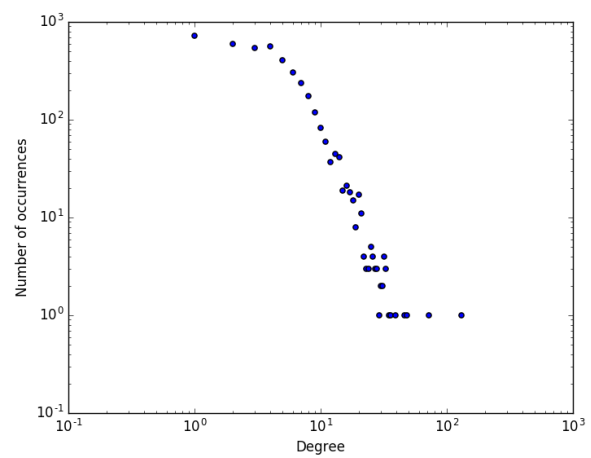

(a) Degree distribution for main validation set

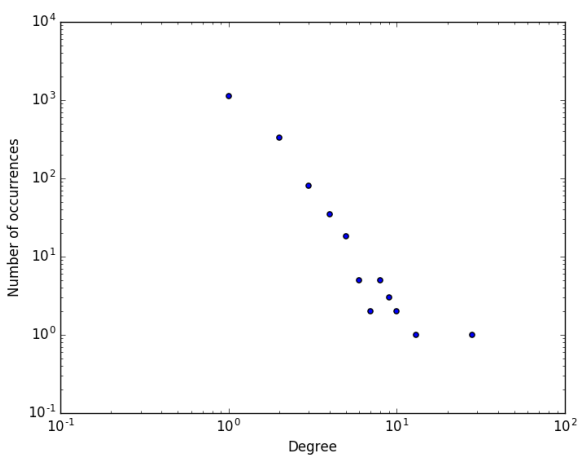

(b) Degree distribution for secondary validation set

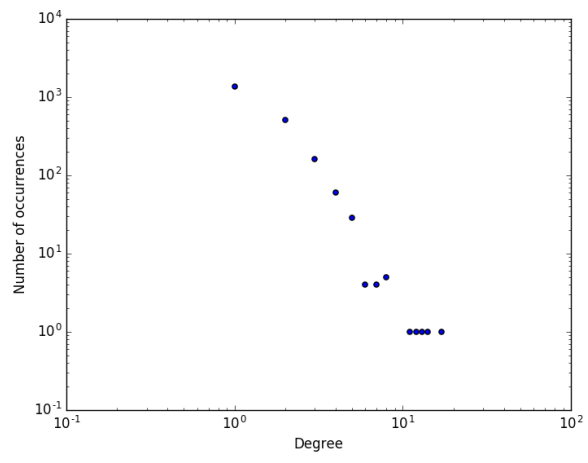

(c) Degree distribution for test set

Figure 1: Degree Distributions for confusion graphs. In these graphs the vertices are classes and the edges are misclassifications made by QANTA.

In order to simplify computation, the graph includes only answers that were confused with at least one other, as the rest would just be isolated vertices. We built these graphs for two of our validation sets, and our test set. The question/answer data that comprises these sets is unfortunately proprietary, so we cannot show examples of question/answer pairs here. Refer to the paper (Iyyer et al., 2014) for an example.

\subsection{The Giant Component}

Recall that our goal is to form groups of answers that are highly confused with each other. An optimistic plan would be to simply find the components of the graph, and split each one into a pair of clusters. Unfortunately, it turns out that QANTA's confusion graph for the primary validation set has very few components. Specifically, 4032 of the 4091 nodes in the graph are in a single, large component.

The reason for the emergence of a giant component is that there are certain answers (vertices) that have extremely high degrees, i.e. the degree distri- bution of these graphs is heavy-tailed. The degree distributions of each graph are shown in Figure 1. The existence of these high degree nodes leads to connectivity between seemingly very different answers.

For example, in the validation set, the most confused answer was the play: Angels in America: A Gay Fantasia on National Themes. It was confused with 131 different answers ranging from the number eight, to Milk, to ASCII. This shows that the errors made on this validation set did not solely include answers that are very similar.

\subsection{Diameter and Neighborhoods}

We now examine some of the other properties of the giant component of this graph. The diameter (i.e. maximum length of a shortest path between two vertices) of the component is 14 . This means that every answer can be reached from every other, along a relatively short path. As an example, one of the few answer with an eccentricity of 14 is "Ribosome". It 


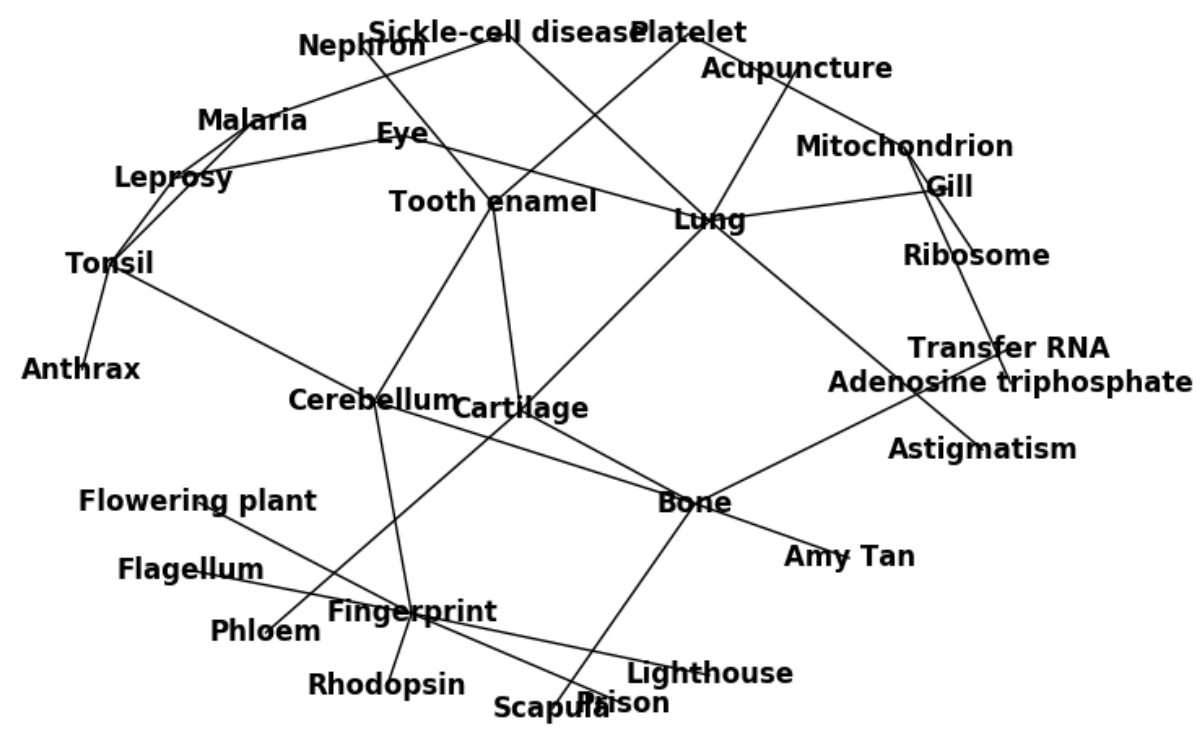

Figure 2: Answers within a short distance of the answer "Ribosome" where edges represent QANTA answering one when the other should be answered.

is a distance of 14 away from the novel Ragtime, via the path:

Ribosome $\rightarrow$ Mitochondrion $\rightarrow$ Platelet $\rightarrow$ Tooth enamel $\rightarrow$ Cartilage $\rightarrow$ Lung $\rightarrow$ Acupuncture $\rightarrow$ Yoga $\rightarrow$ Caste system in India $\rightarrow$ Jacques Cartier $\rightarrow$ HMS Beagle $\rightarrow$ Thor Heyerdahl $\rightarrow$ Ragtime $\rightarrow$ Ragtime (novel).

So at least one question that should have been answered Ribosome was answered as Mitochondrion (or vice versa), the same for Mitochondrion and Platelet, and so on. This is a very characteristic example of the paths connecting examples. Typically, most of the errors are between related answers, but there will be some jump which switches the topic abruptly. Here, the first few answers in the path are biology related, Acupuncture is used as a medical treatment, and yoga comes from India. The connections Caste System in India $\rightarrow$ Jacques Cartier and Thor Heyerdahl $\rightarrow$ Ragtime, however, are not expected.

A subgraph of the confusion graph, consisting of all nodes within a distance of 6 from Ribosome is shown in Figure 2. Aside from for some exceptions at maximal distance (e.g. Lighthouse and Amy Tan), all of the nodes are biology related.

The neighborhood of the answer Bessel Function is shown in Figure 3. Note that here is an overlap with the previous neighborhood via the node Astigmatism. In this neighborhood we see a small bunch of computing and mathematical terms, as well as some whose presences again escape an immediate explanation (e.g. Bhagavad Gita).

This exploration hints that although the graph is essentially one component there are clusters of related answers which are confused with each other

\section{Clustering Classes}

We would like to find clusters of related answers, as in the previous section, but in an algorithmic way. To this end, we run average linkage agglomerative clustering (Murtagh, 1983) on the graph, using the weights defined in the previous section.

Average-link clustering on a confusion graph proceeds by first initializing a singleton cluster for each class. Then, at each step, the two clusters, $C_{i}, C_{j}$ are selected such that they have largest average weight 


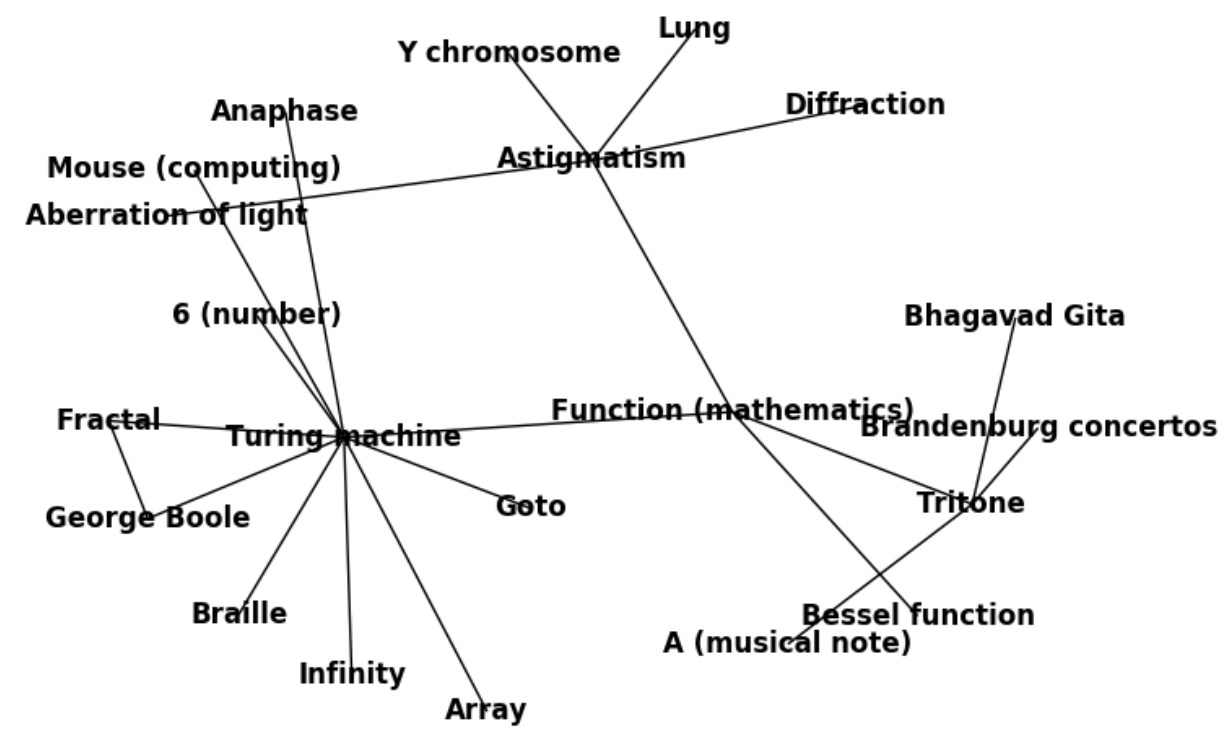

Figure 3: Answers within a short distance of the answer "Ribosome" where edges represent QANTA answering one when the other should be answered.

between them, i.e.:

$$
i, j=\underset{i, j}{\operatorname{argmax}} \frac{1}{\left|C_{i}\right|\left|C_{j}\right|} \sum_{u \in C_{i}} \sum_{v \in C_{j}} w(u, v)
$$

where $w(u, v)$ is the weight of the edge $(u, v)$ if it exists, or 0 otherwise. These clusters are then merged into a single cluster, and the process is repeated until all nodes are in a single cluster. There are 4091 nodes in the graph, so there will be 4090 joining steps. Average-linkage clustering is $O\left(N^{2} \log (N)\right)$, so this computation will become difficult for much larger class sets.

In the context of a confusion graph, two clusters being merged indicates that the classes in the clusters are highly confused. In order to understand our errors better, we examine these clusters. It turns out that many of them are a single answer being added to a larger cluster. To filter out these, we only look at pairs of at least a minimum size, $s$ and having a maximum difference of $d$, i.e. $C_{i}$ and $C_{j}$ such that $\min \left(\left|C_{i}\right|,\left|C_{j}\right|\right) \geq s$ and ||$C_{i}|-| C_{j}|| \leq d$.

When $s=8$ and $d=1$, we found 46 pairs of clusters. Examples are shown in Tables 1 and 2.
Cluster 1 of Table 1 seems highly related to high energy physics and cosmology, whereas Cluster 2, while similar looks related to a different branch of physics with a dash of chemistry. Cluster 1 of Table 2 has many quantities (e.g. energy and enthalpy), with a few other technical terms thrown in. Cluster 2 has a few units and technical terms, but also Leibniz, who is understandably related to science. Unlike the previous cluster pair, there are also some totally unrelated classes: the author Kingsley Amis and his novel Lucky Jim, and the novel The Ox-Bow Incident.

Some clusters fail to be remotely interpretable, but a large number are of similar quality to the ones shown here: Largely related, with a few outliers thrown in. These clusters give us a tractable way of interpreting the confusion of our classifier.

\section{Cluster Analysis with Autoslog}

Autoslog is a system which find repeated patterns of parts of speech in a corpus of documents. An example of such a pattern is "Battle of iNoun phrase $i$ ". For each cluster developed in the previous section, 


\begin{tabular}{c|c} 
Cluster 1 & Cluster 2 \\
\hline Theory of Relativity & Pauli Exclusion Principle \\
Time Dilation & Alloy \\
Reynolds Number & Electronegativity \\
Cosmological Constant & Bessemer Process \\
Sunspot & Nuclear Fission \\
Muon & Hydrogen Peroxide \\
Speed of Light & Fraunhofer lines \\
Kinetic Energy & Palladium \\
Dark Energy & Platinum \\
& Hund's Rules
\end{tabular}

Table 1

\begin{tabular}{c|c} 
Cluster 1 & Cluster 2 \\
\hline Quicksort & Tachyon \\
Hyperbola & Monad \\
Work (physics) & Time \\
Fault (geology) & Kingsley Amis \\
Energy & The Ox-Bow Incident (Novel) \\
Variance & Gravitational Constant \\
Frequency & Lucky Jim \\
Median & Mass \\
Standard Deviation & Gottfried Wilhelm Leibniz \\
Enthalpy & Kilogram \\
Circle & Ossian \\
Scattering &
\end{tabular}

Table 2

Clusters of answers which QANTA confuses with each other often.

we use the set of questions mapping to an answer in the cluster as a corpus, and run Autoslog on it. We then filter to patterns which occur much more frequently in that correspond to one set of questions but not the other.

For example, in Cluster 1 of Table 1, the pattern "[Verb phrase: be] [Adjective Phrase: equal]" was found. It matches any verb phrase with the root verb of "to be" followed by an adjective phrase with the root of "equal". This pattern should match statements about the various constants and quantities found in the cluster.

For another pair of clusters in which one is composed mostly of nations and locations, and the other is mostly chemicals, we find the patterns "[Noun phrase: capital] of" and "[Noun phrase: Nation] with". However, the pattern "[Verb phrase: Named] for" occurs about equally as much in each cluster. Such patterns may be part of what contributes to the classifier confusing the clusters.

\section{Future Work}

We have developed and examined confusion graphs for our classifier, but not yet applied them to improving its performance. We would like to add the patterns found by Autoslog as features for QANTA, and evaluate its increase in performance. If this is successful, we can again build a confusion graph, find patterns and add them as features, and repeat the process. This is a sort of automatic feature engi- neering. How much performance improvement we can get out of this method is an open question.

\section{Conclusion}

Confusion graphs can help us understand the nature of the errors occurring in a classifier when the number of classes is very large. We have applied this to examine the errors of the question answering system, QANTA. From the graph, we derive clusters of answers which are easily confused, and find textual features which discriminate between the clusters.

\section{References}

Mohit Iyyer, Jordan Boyd-Graber, Leonardo Claudino, Richard Socher, and Hal Daumé III. 2014. A neural network for factoid question answering over paragraphs. In Empirical Methods in Natural Language Processing.

Fionn Murtagh. 1983. A survey of recent advances in hierarchical clustering algorithms. The Computer Journal, 26(4):354-359.

Ellen Riloff. 1996. Automatically generating extraction patterns from untagged text. In Proceedings of the national conference on artificial intelligence, pages 1044-1049. AAAI Press. 